\title{
Clinical Significance of Lenticulostriate Vasculopathy
}

\author{
Chaw-Liang Chang, MD ${ }^{1,2,3}$ \\ ${ }^{1}$ Department of Pediatrics, Cathay General Hospital, Hsinchu, Taiwan \\ ${ }^{2}$ Center for Medical Education and Research, Cathay General Hospital, Hsinchu, Taiwan \\ ${ }^{3}$ School of Medicine, Fu-Jen Catholic University, New Taipei City, Taiwan \\ juliancsr@yahoo.com.tw
}

\begin{abstract}
Lenticulostriate vasculopathy $(L S V)$ is a common finding on routine cranial ultrasound examinations. However, the clinical meaning of LSV remains unclear, more than 3 decades after the first LSV was reported. This review examined the literature in an attempt to clarify the clinical significance of LSV and provide practical guidelines for daily practice. LSV has been linked to various etiologies including cytomegalovirus infection, which has been studied the most among all infections associated with LSV. Infants with isolated LSV generally have normal neurodevelopment and a favorable long-term prognosis. However, various clinical conditions are associated with LSV. This paper also reviews the clinical correlations between LSV and associated disorders including neurodevelopment, neuropsychiatric disorders, and infantile basal ganglia stroke.
\end{abstract}

Keywords: Lenticulostriate vasculopathy, ultrasound, infant, neonate, stroke, cytomegalovirus

\section{INTRODUCTION}

Lenticulostriate vasculopathy (LSV) is a term given to "bright" or "hyperechogenic" vessels in the region of the thalamus and basal ganglia that are observed on cranial ultrasonograms in neonates. ${ }^{1,2}$ An LSV was first observed more than 3 decades ago; the term "vasculopathy" was first used by Teele et al in 1988. ${ }^{1} \mathrm{LSV}$ is a common finding on neonatal cranial ultrasonograms. The reported incidence of LSV ranges from $0.3 \%$ to $32 \%$, depending on the population studied. ${ }^{2-4}$ Because of the technological advances in cerebral ultrasound imaging and growing awareness among doctors of this cerebral ultrasound finding, the incidence of LSV identification has increased since the finding was first recognized. ${ }^{2,5}$ The frequencies of transducers used for neonatal cerebral ultrasound 3 decades ago were 5-7.5 MHz, whereas the frequencies of contemporary neonatal cerebral ultrasound transducers range from 10 to $15 \mathrm{MHz}$, which greatly amplifies echogenicities within the cerebral tissue. ${ }^{6}$

Although LSV is not a rare finding on cranial ultrasound examination, and despite cranial ultrasound examinations having become a routine examination for neonates admitted to intensive care units, the clinical meaning of LSV remains unclear, and much remains to be understood. Several reports have suggested that LSV may be a benign phenomenon observed in otherwise healthy infants. ${ }^{3,5,7}$ However, various clinical conditions have been associated with LSV. Although large-scale studies on LSV are limited, this review examined the literature to attempt to clarify the clinical significance of LSV. 


\section{LSV AND INFECTION}

LSV has been linked to various infectious etiologies including cytomegalovirus (CMV), rubella, amniotic infection, Streptococcus sepsis, and syphilis. ${ }^{1,8-10}$ Among those infections associated with LSV, CMV infection has been studied the most. The first observed LSV, reported by Grant et al in 1985, was in a 1240-g second-born twin with congenital CMV infection. ${ }^{11}$ Studies have shown a low yield of routine toxoplasmosis, rubella, cytomegalovirus, and herpes (TORCH) screenings in neonates with LSV. ${ }^{12,13}$ None of 38 infants with LSV had a positive CMV urine culture test in a study by De Jong EP et al. ${ }^{13}$

In contrast to the aforementioned research, more recent studies, including 2 in 2015, have reported a strong correlation between CMV infection and LSV. In a study by Amir et al, LSV was detected on the initial brain ultrasounds of $54.3 \%$ of infants diagnosed with congenital CMV infection. ${ }^{14}$ The authors concluded that LSV was a possible marker of high risk of sensorineural hearing loss in infants with congenital CMV infection ${ }^{14}$; this conclusion was supported by later studies. ${ }^{15,16}$ Hong et al categorized LSV cases as mild or severe; they found that LSV, particularly severe LSV, is a predictor of CMV infection. ${ }^{16}$ The CMV detection rate using urine polymerase chain reaction was $69 \%$ in the severe LSV group and $23 \%$ in the mild LSV group. ${ }^{16}$

\section{OTHER NONINFECTIOUS CONDITIONS ASSOCIATED WITH LSV}

Various perinatal and neonatal conditions have been reported to be associated with LSV including trisomy 13 syndrome, Down syndrome, hypoxic ischemic state, hyperbilirubinemia, cardiac disease, fetal alcohol syndrome, congenital malformations, respiratory distress syndrome, neonatal lupus erythematosus, twin-to-twin transfusion, sialidosis, hydrops fetalis, and diabetic fetopathy. ${ }^{1,9,10,12,17,18}$ The associations of these conditions with LSV are based on case reports or studies of small sample sizes; therefore, the relationships between LSV and these conditions require further assessment in large-scale studies.

In a prospective randomized study by Mittendorf et al, women in preterm labor were randomized to receive either magnesium sulfate or other tocolytics (149 women gave birth to 165 babies). ${ }^{19}$ They found that exposure to $\geq 50 \mathrm{~g}$ of tocolytic $\mathrm{MgSO} 4$ was significantly associated with the development of LSV. ${ }^{19}$ Additional large-scale studies are necessary to confirm this observation.

\section{LSV AND NEURODEVELOPMENT}

Infants with isolated LSV generally have normal neurodevelopment and a favorable long-term prognosis. ${ }^{5,7}$ In a study by El Ayoubi et al, 53 infants with LSV were followed for 2 to 9 years; 35 $(66 \%)$ were found to be developmentally normal at the end of follow-up, whereas $8(15 \%)$ had minor deviations, 4 (7.5\%) had moderate deviations, and 6 (11.3\%) had major neurodevelopmental deficits. Of the 34 infants with isolated LSV, 27 (79\%) were normal, compared with 8 of the 19 (42\%) infants with LSV and an associated condition. ${ }^{7}$

In a different study, premature infants with LSV exhibited lower scores for neurodevelopment and, in particular, cognitive and behavioral performance. ${ }^{20}$ Most infants with LSV and developmental delay had an associated disease such as CMV infection, major malformations, fetal alcohol exposure, neonatal hypoxia, and systemic Streptococcus sepsis. ${ }^{4,5,18}$ 
Shin et al categorized LSV as being low grade (1 or 2 thin branches) and high grade (3 or more prominent branches). They determined that high-grade LSV and absent color Doppler flow on lenticulostriate vessels were significantly associated with neurodevelopmental delay. ${ }^{4}$

\section{LSV AND NEUROPSYCHIATRIC DISORDERS}

Wang and Kuo followed 34 children with LSV and evaluated them at 7 to 9 years of age. Twenty-eight of the children $(82 \%)$ had one or more neuropsychiatric disorders including tics, attention deficit hyperactivity disorder, obsessive-compulsive disorder, neurological deficits, and developmental delay. ${ }^{21}$ They categorized the infants with LSV into 3 groups: with perinatal etiology (perinatal group; 16 patients), with acquired disorder (acquired group; 5 patients), and with other unknown etiologies (idiopathic group; 13 patients). Wang and Kuo found that the rates of neuropsychiatric disorders were higher in the idiopathic group (54\%) than in the perinatal and acquired groups (10\%); similar results were found for attention deficit hyperactivity disorder (54\% vs. $10 \%$ ), tics (38\% vs. $10 \%$ ), and obsessive-compulsive disorder (13\% vs. 5\%). By contrast, compared with the perinatal and acquired groups, the idiopathic group exhibited lower rates of mortality (33\% vs. $0 \%$ ), developmental delay ( $24 \%$ vs. $8 \%$ ), mental retardation (24\% vs. $0 \%$ ), and neurological deficits $(29 \%$ vs. $0 \%){ }^{21}$ Because only one study focused on the long-term outcome of LSV and neuropsychiatric disorders, this conclusion must be confirmed by large-scale studies.

\section{LSV AND INFANTILE BASAL GANGLia STROKE}

Teele et al demonstrated in their postmortem neuropathologic examination that the echogenicities observed in a cranial ultrasonogram indicate the presence of basophilic deposits (ie, mineralization) or hypercellular lesions within the arterial walls. ${ }^{1}$ They referred to this condition as "mineralizing vasculopathy."1

The mineralization of lenticulostriate arteries made them more rigid and vulnerable to shear injury compared with normal vessels; even a mild head trauma may result in injury of the arteries. The associated stretching may engender vasospasm and thrombosis, causing ischemic stroke. Infantile basal ganglia stroke after mild trauma is a rare type of stroke that clearly implicates lenticulostriate calcification as the pathological substrate. ${ }^{22-27}$ Nevertheless, the mineralization of LSV was not observed in all neonatal brains examined histologically, ${ }^{9,28}$ suggesting that not all neonates with LSV have a mineralizing vasculopathy and not all neonates with LSV are at risk of infantile basal ganglia stroke.

\section{CONClusion}

LSV is a common clinical finding in cerebral ultrasonograms and a nonspecific marker of previous insult to the developing brain. Most neonates and infants with low-grade LSV have normal neurodevelopment. If mild LSV is detected incidentally in otherwise healthy neonates, normal development can be expected and regular ultrasound follow-up for LSV is adequate. Study to identify congenital CMV infection is recommended for high-grade LSV. If LSV combined with CMV infection is confirmed or if another disease state is recognized, further examination (eg, a hearing test) according to the associated condition is necessary. Although the incidence of infantile basal ganglia 
stroke is low, if the LSV is high-grade (multiple branches with high echogenicity), the parents should be informed about the risk of infantile basal ganglia stroke, with particular caution taken to prevent such infants falling from beds or other high places.

\section{REFERENCES}

[1] Teele RL, Hernanz-Schulman M, Sotrel A. Echogenic vasculature in the basal ganglia of neonates: a sonographic sign of vasculopathy. Radiology. 1988;169(2):423-7.

[2] Sisman J, Rosenfeld CR. Lenticulostriate vasculopathy in neonates: Is it a marker of cerebral insult? Critical review of the literature. Early Hum Dev. 2015;91(7):423-6.

[3] [Leijser LM, Steggerda SJ, de Bruine FT, et al. Lenticulostriate vasculopathy in very preterm infants. Arch Dis Child Fetal Neonatal Ed. 2010;95(1):F42-6.

[4] Shin HJ, Kim MJ, Lee HS, Namgung R, Park KI, Lee MJ. Imaging patterns of sonographic lenticulostriate vasculopathy and correlation with clinical and neurodevelopmental outcome. $J$ Clin Ultrasound. 2015;43(6):367-74.

[5] Shen EY, Weng SM, Kuo YT, Chiu NC, Ho CS. Serial sonographic findings of lenticulostriate vasculopathy. Acta Paediatr Taiwan. 2005;46(2):77-81.

[6] Koral K, Sisman J, Pritchard M, Rosenfeld CR. Lenticulostriate vasculopathy in neonates: Perspective of the radiologist. Early Hum Dev. 2015;91(7):431-5.

[7] El Ayoubi M, de Bethmann O, Firtion G, Monset-Couchard M. [Neonatal lenticulostriate vasculopathy. Mid and longterm outcome (70 cases)]. Arch Pediatr. 2004;11(1):18-23. French.

[8] Ben-Ami T, Yousefzadeh D, Backus M, Reichman B, Kessler A, Hammerman-Rozenberg C. Lenticulostriate vasculopathy in infants with infections of the central nervous system sonographic and Doppler findings. Pediatr Radiol. 1990;20(8):575-9.

[9] Hughes P, Weinberger E, Shaw DW. Linear areas of echogenicity in the thalami and basal ganglia of neonates: an expanded association. Work in progress. Radiology. 1991;179(1):103-5.

[10] Weber K, Riebel T, Nasir R. Hyperechoic lesions in the basal ganglia: an incidental sonographic finding in neonates and infants. Pediatr Radiol. 1992;22(3):182-6.

[11] Grant EG, Williams AL, Schellinger D, Slovis TL. Intracranial calcification in the infant and neonate: evaluation by sonography and CT. Radiology. 1985;157(1):63-8.

[12] El Ayoubi M, de Bethmann O, Monset-Couchard M. Lenticulostriate echogenic vessels: clinical and sonographic study of 70 neonatal cases. Pediatr Radiol. 2003;33(10):697-703.

[13] de Jong EP, Lopriore E, Vossen AC, et al. Is routine TORCH screening warranted in neonates with lenticulostriate vasculopathy? Neonatology. 2010;97(3):274-8.

[14] Amir J, Schwarz M, Levy I, Haimi-Cohen Y, Pardo J. Is lenticulostriated vasculopathy a sign of central nervous system insult in infants with congenital CMV infection? Arch Dis Child. 2011;96(9):846-50.

[15] Bilavsky E, Schwarz M, Pardo J, et al. Lenticulostriated vasculopathy is a high-risk marker for hearing loss in congenital cytomegalovirus infections. Acta Paediatr. 2015;104(9):e388-94. 
[16] Hong SY, Yang JJ, Li SY, Lee IC. Lenticulostriate Vasculopathy in Brain Ultrasonography is Associated with Cytomegalovirus Infection in Newborns. Pediatr Neonatol. 2015;56(6):408-14.

[17] Coley BD, Rusin JA, Boue DR. Importance of hypoxic/ischemic conditions in the development of cerebral lenticulostriate vasculopathy. Pediatr Radiol. 2000;30(12):846-55.

[18] Makhoul IR, Eisenstein I, Sujov P, et al. Neonatal lenticulostriate vasculopathy: further characterisation. Arch Dis Child Fetal Neonatal Ed. 2003;88(5):F410-4.

[19] Mittendorf R, Kuban K, Pryde PG, Gianopoulos JG, Yousefzadeh D. Antenatal risk factors associated with the development of lenticulostriate vasculopathy (LSV) in neonates. $J$ Perinatol. 2005;25(2):101-7.

[20] Chamnanvanakij S, Rogers CG, Luppino C, Broyles SR, Hickman J, Perlman JM. Linear hyperechogenicity within the basal ganglia and thalamus of preterm infants. Pediatr Neurol. 2000;23(2):129-33.

[21] Wang HS, Kuo MF, Chang TC. Sonographic lenticulostriate vasculopathy in infants: some associations and a hypothesis. AJNR Am J Neuroradiol. 1995;16(1):97-102.

[22] Ivanov I, Zlatareva D, Pacheva I, Panova M. Does lenticulostriate vasculopathy predipose to ischemic brain infarct? A case report. J Clin Ultrasound. 2012;40(9):607-10.

[23] Landi A, Marotta N, Mancarella C, Marruzzo D, Salvati M, Delfini R. Basal ganglia stroke due to mild head trauma in pediatric age - clinical and therapeutic management: a case report and 10 year literature review. Ital J Pediatr. 2011;37:2.

[24] Deveber G. Stroke in infancy: a convergence of causes. Dev Med Child Neurol. 2014;56(1):9-10.

[25] Lingappa L, Varma RD, Siddaiahgari S, Konanki R. Mineralizing angiopathy with infantile basal ganglia stroke after minor trauma. Dev Med Child Neurol. 2014;56(1):78-84.

[26] Yang FH, Wang H, Zhang JM, Liang HY. Clinical features and risk factors of cerebral infarction after mild head trauma under 18 months of age. Pediatr Neurol. 2013;48(3):220-226.

[27] Yang ST, Lee WT, Hung KL, Chang CL. Infantile basal ganglia stroke after mild head trauma. Neurology. 2015;84(23):2381-2382.

[28] Cabanas F, Pellicer A, Morales C, Garcia-Alix A, Stiris TA, Quero J. New pattern of hyperechogenicity in thalamus and basal ganglia studied by color Doppler flow imaging. Pediatr Neurol. 1994;10(2):109-116. 